\title{
COMPARATIVE LABORATORY EVALUATION OF DIMENSIONAL ACCURACY FOR 3D-PRINTED COMPLETE MAXILLARY DENTURE
}

\author{
Hala Mohamed AbdElHameed* and Sara Zaky Mohamed**
}

\begin{abstract}
Purpose: The objective of the research was to investigate dimensional accuracy concerning denture teeth deviation and vertical dimensional changes for three-dimensional printed, monolithic dentures relative to heat-cured, acrylic resin dentures.

Materials and methods: A waxed-up reference maxillary denture was constructed on an educational master cast, and then duplicated to produce ten simulated waxed-up dentures. The planned virtual denture was designed by subtracting the scanned cast from the scanned waxed-up denture with the cast. This virtual denture was considered as a reference and saved alone in 3DStandard Tessellation Language (STL) format. From this reference (CAD maxillary denture) ten DLP dentures were fabricated. Each fabricated heat-cured, and 3D-printed denture was scanned separately, and the scanned data was saved in an STL file. Each STL file of scanned maxillary dentures was superimposed on a reference virtual maxillary denture to evaluate the degree of denture teeth deviation. An electronic digital caliper was used to measure the occlusal vertical dimension (OVD) of the waxed-up denture and after denture fabrication for both groups to evaluate the change in OVD.
\end{abstract}

Results: A paired t-test was used to compare the recorded data between both groups. It was reported that the higher mean values for either denture teeth deviation or vertical dimensional changes after dentures fabrication were noticed in heat-cured acrylic resin dentures. These values were highly statistically significant between the tested groups for both items of investigations at $\mathrm{p} \leq 0.01$.

Conclusion: Under the restrictions of this research, tooth movements occur to some degree in both surveyed groups. However, three-dimensional monolithic printing dentures displayed greater dimensional precision with respect to denture teeth deviation and vertical dimensional alteration compared to heat-curing acrylic resin.

KEYWORDS: Tooth movement - dimensional accuracy - complete denture - 3D-printed dentures

Associate Professor of Prosthodontics, Faculty of Dentistry, Suez Canal University

** Lecturer of Prosthodontics, Faculty of Dentistry, Suez Canal University 


\section{INTRODUCTION}

Edentulism has a high level of concern for most people, and replacing them with artificial replacements as dentures is essential for the continuation of a healthy life. One of the dentures' primary duties is their ability to function effectively over the required period. ${ }^{1}$

Polymethyl methacrylate (PMMA) has almost been the widely used denture base material since its foundation. Significant characteristics include good aesthetic features, appropriate strength, substantially less toxicity, repair capabilities, ease of handling, and processing technology. However, PMMA-based dentures experience inevitable dimensional changes during processing, heat expansion, cooling contraction and polymerization shrinkage. ${ }^{2}$

Ideally, the established occlusion in a trial denture should be preserved through the process of polymerization. However, shrinkage of denture base resin during polymerization is an indelible issue affecting the denture teeth' location, hence the final denture occlusion. ${ }^{3,4}$

In acrylic resin flasking strategy, strain-accompanied by stress release during flaking can cause linear changes. These variations allow for artificial movements of the teeth, leading to alterations in the occlusal vertical dimension. ${ }^{5,6}$

Today, with the development of dental material and technology, the feasibility of denture construction using computer-aided design/ computer-aided manufacturing (CAD / CAM) technology has been demonstrated by numerous published studies. The additives manufacturing through $3 \mathrm{D}$ printers has become a prime concern because of their ability to mold a variety of materials in the field of prosthetic dentistry based on CAD data. ${ }^{7}$

Additive manufacturing undertakes the design of the product from a 3D file imported in the Standard Tessellation Language (STL) format, which segments CAD drawings into tiny thin sections and enables layer by layer processing. It can deliver parts without the need for a tool or mold. Compared to subtractive manufacturing technologies such as milling, computer numerical controlled (CNC) machine, additive manufacturing has the advantage of high flexibility and diminished material wastage. ${ }^{8}$

Such technology is also termed rapid prototyping system (RPS). 3D printing is a relatively modern technology, RPS is designed to build solid and precise 3D physical models capable of replicating accurately complex anatomical structures derived from computer data. ${ }^{9}$

Combining scanned information on dental structures and the virtual design of final prosthetic reconstructions (CAD) with an ultraviolet (UV) laser beam passed over a photosensitive resin, producing an accurate $3 \mathrm{D}$ dental prosthesis characterized by a complete replica of the external surface and internal structure. ${ }^{10}$

The objective of this in-vitro study is to compare denture teeth deviation and vertical dimensional changes of simulated complete maxillary dentures constructed utilizing the compression molding heatcured technique with (RPS) 3D printing technology. The null hypothesis is that no difference will be found between groups.

\section{MATERIALS AND METHODS}

To find a significant difference between the groups, a sample size of approximately ten was required, this based on the results already reported. ${ }^{11}$

Consequently, a sample size of 10 for each group was evaluated to be acceptable. Eleven maxillary master stone castings (one of which was used for the construction of the reference model) were obtained by pouring hard dental stone type III (Snow Rock, Korea) into a standardized, ready-made silicon mold of completely edentulous maxillary arch free from undercuts. Indexing of the base of each stone cast was performed to enable denture remounting on the articulator. 
On a semi-adjustable articulator (Quick Master, France), one cast was mounted with its occlusion block. Eleven identical sets of appropriate sizes of maxillary denture teeth were chosen, and one set of them was used to set up teeth for the mounted master occlusion block. After that, the waxing up of the trial denture was finalized to create a master maxillary waxed-up denture that represented the reference model (Fig.1-a).

\section{Construction of identical waxed-up dentures:}

\section{I-Preparing the master trial waxed up denture jig:}

Duplication of the reference waxed-up denture was performed via a pourable silicone duplicating material (Dupliflex silicones, Protechno, Spain) in a custom plastic flask to create the same denture base contours and teeth positions.

\section{II-Duplication of master trial waxed up denture}

Acustom plastic flask was used for the duplication process. The reference maxillary waxed-up denture with its master cast was housed on the base of a custom flask, so that at least $5 \mathrm{~mm}$ clearance was available all around. Duplicating material was poured under vibration; once it set, the cast with the waxed-up simulated denture were removed. This created fixed silicon mold,in which ten identical waxed-up maxillary dentures were produced.

\section{III-Formation of simulated trial dentures:}

In the silicon mold, an identical set of maxillary denture teeth has been implanted in their respective positions. The cast was secured in its position in the silicon mold, and the molten wax filled the area between the teeth and the cast. Once the wax was dried, the cast with the formed trial denture were carefully removed from the silicon mold. These protocols were repeated until all similar, 10 waxedup trial maxillary dentures were created.

For further verification, that all teeth with simulated waxed dentures were located in the same spatial position and vertical dimension that the maxillary reference waxed-up denture was employed on the articulator; a plaster jig was built on the lower member of the articulator opposing the maxillary reference waxed-up denture. All the maxillary waxed-up dentures were mounted against this jig, and the occlusal surface of the teeth was oriented against the stone matrix until the incisal pin reached the incisal table.

\section{Regarding the strategy used for denture fabrica- tion, there are two researched groups each group consists of 10 samples.}

Group (A): The maxillary waxed-up dentures were fabricated in heat-cured acrylic resin using water bath curing method (long cycle).

Group (B): The maxillary waxed-up dentures were fabricated utilizing additive manufacturing forming a three-dimensional printed resin denture.

\section{Fabrication of three-dimensional printed dentures}

The reference master cast was digitized using a lab scanner (Swing, DOF lab, Korea) followed by another scanning of the reference cast with waxed up denture after spraying opacifier on the waxed-up denture to be detected by the scanner (fig.1-A, B). The 3D-scanned data from both digital scanning was saved in a standard tessellation language file (STL).

The planned virtual denture was designed by subtracting the scanned cast from the scanned cast with waxed-up denture using blender software (Blender, Netherlands) with the aid of Boolean subtract function (fig.1-C). This virtual denture was considered as a reference and saved separately in an STL file, which was then sent to slicer software (Chitubox, China).

From this reference, CAD maxillary denture, 10 maxillary digital light processed (DLP) denture were fabricated. 3D-printable Resin liquid (Dental Sand A2, Harzlabs, Russia) was added to the 3D printer (Photon, Anycubic, China). Printing was started with a build angle of $45^{\circ}$, and printing thickness on the z-axis was set at 50 microns. Supports were 
selected without interrupting the fitting surface of the denture (fig.1-D).

After printing, the supports were removed, and the printed dentures were cleaned in an ultrasonic bath with ethyl alcohol for 10 minutes to remove excess resin.
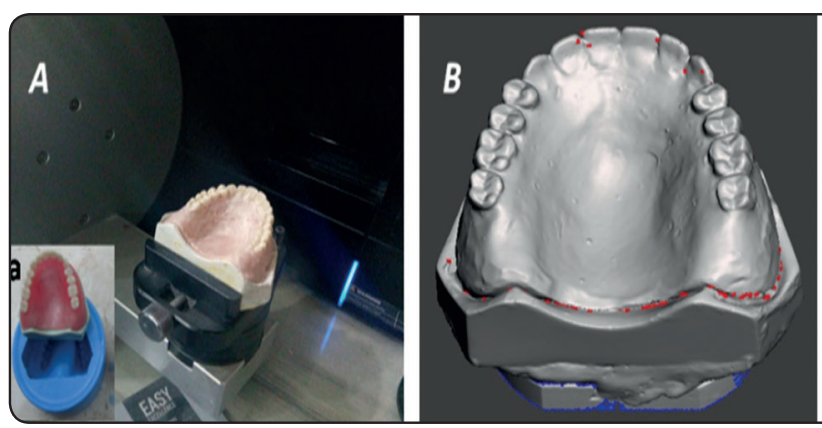

The denture bases were post-polymerized for 40 minutes using an ultraviolet polymerization unit (bre.Lux power Unit 2, Bredent, Germany) as instructed by the manufacturer. Each fabricated heat-cured and 3D-printed denture was scanned separately and was saved as an STL file.

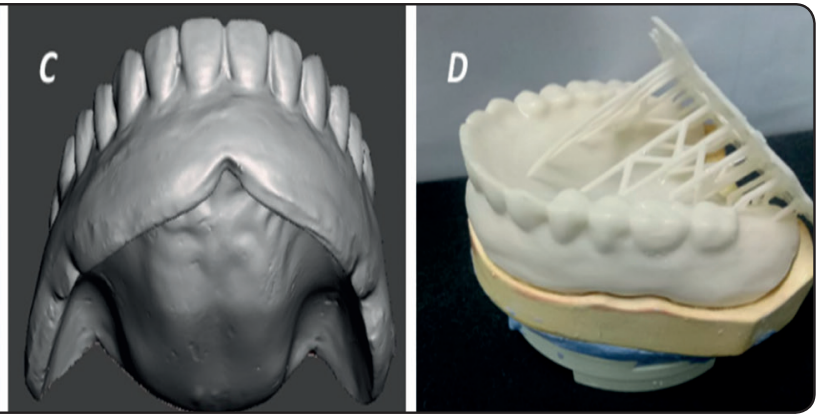

Fig. (1) A) scanning of the waxed-up denture with the reference cast, B) digitized waxed-up denture, C) planned virtual denture by subtracting, D) 3D-printed denture

\section{A-Measuring tooth movements}

Each STL file of the scanned maxillary dentures was superimposed on a reference virtual maxillary denture. To compare the degree of denture teeth deviation, measurements were made using the scanned cast base as the reference, which was started manually using 3 points, followed by final adjustment utilizing the best fit function via the software (gom inspect 2018, GOM, Germany)

Then the deviation of denture teeth was compared to that of reference virtual maxillary denture using inspect deviation function. The colorcoded 3D-surface deviation spectra were set to have a maximum critical value of $\pm 0.8 \mathrm{~mm}$ and visually display the direction and the amount of tooth movement.

Deviation was labeled at the incisal edge and cusp tip for anteriors and at buccal, palatal cusp tips, and marginal ridges for posterior teeth. Surface matching represented by 46 points for each denture. Positive and negative average deviation values were obtained for each specimen (fig 2- A, B).

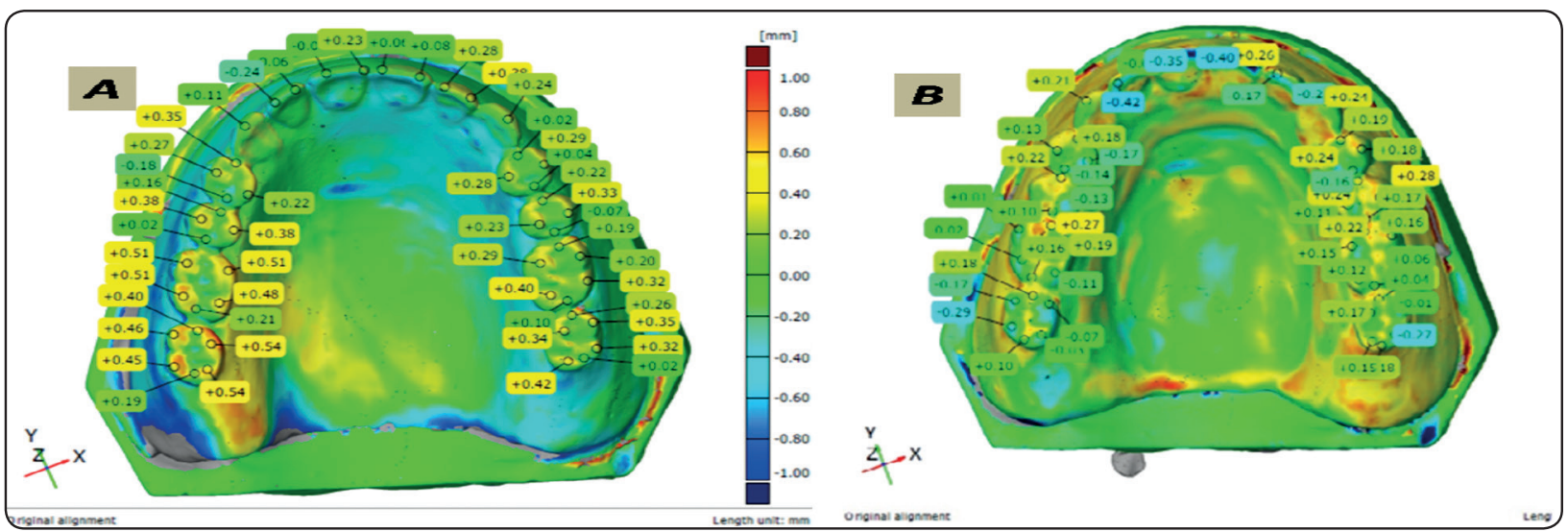

Fig. 2 (A, B): Teeth deviation results for heat-cured and 3D-printed dentures, respectively 


\section{B) Measuring the changes in the vertical dimen- sion of occlusion}

The articulator with its mounted waxed-up denture was established in a steady and fixed designated position on the table of measuring apparatus to guide the position of the articulator concerning all assessments..$^{12}$

The occlusal vertical dimension (OVD) was assessed using an electronic digital caliper (Electronic Digital Caliper, Shan, Japan), with a precision of $0.01 \mathrm{~mm}$ for all test samples. One of the two extremities of the digital caliper was mounted to the measuring device table. So, this extremity was installed under the lower articulator member while the other one was installed at a definite reference mark on the upper articulator member.

The distance between upper and lower members of the articulator before denture fabrication (initial measurement for the waxed up dentures) was measured via the digital caliper. After dentures fabrication of both groups, the maxillary dentures were reoriented to the original mounting of the reference cast on the articulator, and the (final measurement) of the OVD was registered (fig.3-A, B).

The difference between the final and initial measurements indicated changes in the OVD of both groups. These records were estimated to the nearest $0.01 \mathrm{~mm}$ and were statistically analyzed.
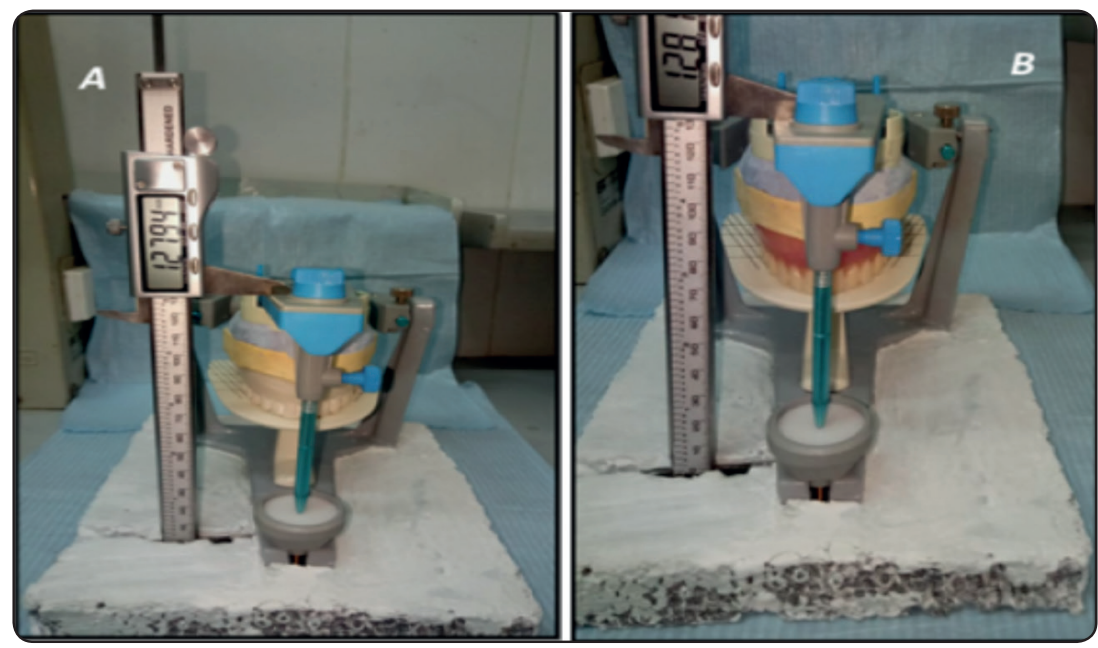

Fig. 3 (A, B): Measuring vertical dimension changes for 3D-printed and heat-cured dentures respectively using an electronic digital caliper.

\section{RESULTS}

The mean values of denture teeth deviation and vertical dimensional changes for all dentures of both studied groups were recorded and statistically analyzed. Data were described as means and standard deviation. The paired t-test compared the reported data between both groups. The significance level was set at $\mathrm{p} \leq 0.05$, and highly significant at $\mathrm{p} \leq 0.01$.

The table and diagrams below reveal that the highest mean values for denture teeth deviation and or vertical dimension changes after denture manufacturing were detected in the pack and pressed hot water bath heat-cured acrylic resin denture than the three-dimensional printed one.

These values were statistically highly significant between tested groups for both items of investigations at $\mathrm{p} \leq 0.01$

The Following Table shows the mean values (in $\mathrm{mm}$ ) for denture teeth deviation and changes in the vertical dimension for both types of dentures 


\begin{tabular}{|c|c|c|c|}
\hline & & Denture teeth deviation & Vertical dimension changes \\
\hline Heat-cured denture & Mean \pm SD & $0.188 \pm 0.07$ & $0.584 \pm 0.18$ \\
\hline 3D-printed denture & Mean \pm SD & $0.043 \pm 0.02$ & $0.36 \pm 0.1$ \\
\hline & $\mathrm{P}$ & $0.000^{* *}$ & $0.002^{* *}$ \\
\hline
\end{tabular}

$S D=$ Standard deviation *significant at $p \leq 0.05$

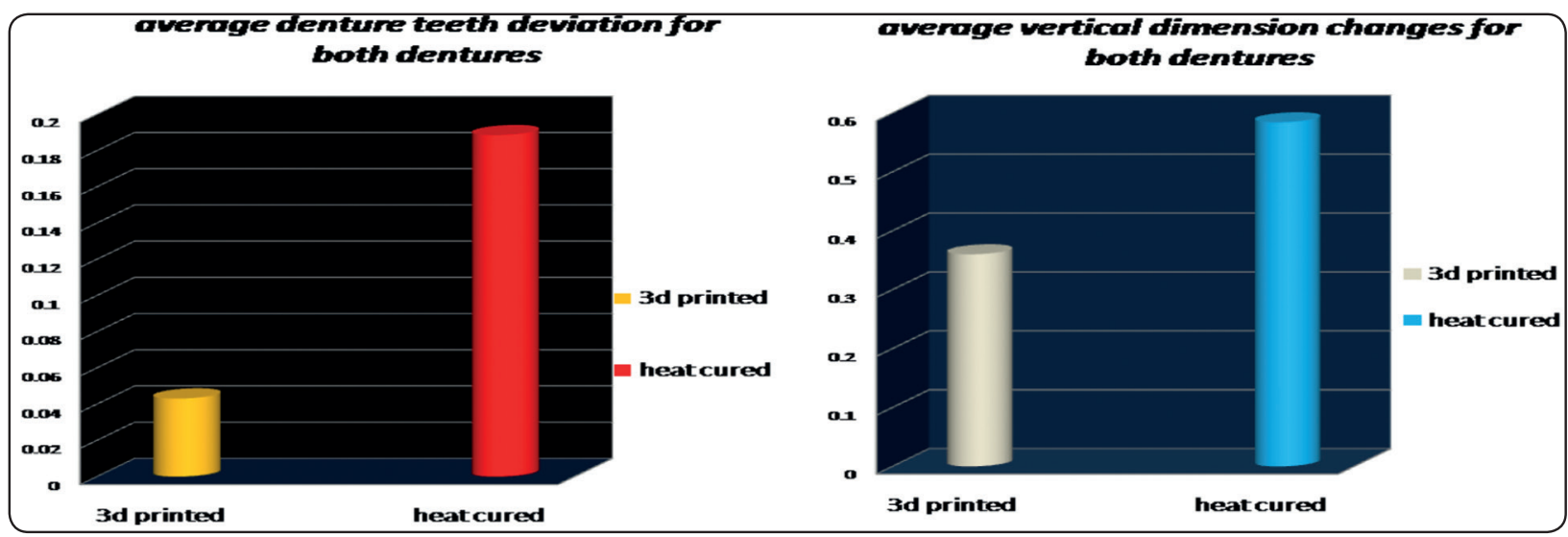

Fig. (4)

\section{DISCUSSION}

The number of patients with edentulous jaw has been over growing with the growth of the world population. The most common treatment is full dentures and, since its introduction in 1937, polymethyl methacrylate (PMMA) has been principally the preferred material used in denture manufacturing. It has numerous advantages such as an excellent aesthetic characteristic, low water sorption and solubility, low toxicity, simplicity of repair, and an uncomplicated molding processing technique. Likewise, it has adequate strength and appropriate physical properties. Therefore, acrylic resins believed to be the gold standard for denture fabrication. ${ }^{13,14}$

Profit of complete denture management relies upon distinct factors, including adaptation, physiological vertical dimension, proper condylar location, lack of occlusal disturbance, emotional and aesthetic aspects. ${ }^{15}$

Occlusal changes occurred during denture processing may contribute to intervention that can cause traumatic occlusion, asymmetric transmission of occlusal stresses to the tissue, and loss of comfort and masticatory efficiency that adversely affect the oral function of the dentures. Teeth can be a key factor in denture distortion since a rise in vertical dimension could be correlated with a few changes in tooth position. ${ }^{16}$

Throughout the production of full dentures, multiple laboratory factors may result in a change in the occlusion. Such factors are linked to the inherited features of materials and procedures, as well as possible extrinsic mistakes made by a dental technician or a dentist. Regarding the processing technique, the movement of the teeth and the opening of the incisal pin can occur with a subsequent increase in the vertical dimension of occlusion.$^{17}$

Various researches were conducted to recognize surpassing materials and processing techniques to minimize occlusion changes. Dental laboratories are progressively turned towards integrating digital formats and procedures, including 3D scanner, CAD, and various technologies for additive manufacture. The 3D scanner captures data from a patient's unique dental anatomy converting it to a digital 3D 
CAD file. This file is an editable/printable file, using photosensitive liquid resins, repeatedly layered on a support structure, and polymerized by functional additive manufacturing technologies through an ultraviolet or visible light source. ${ }^{18}$

Regarding the results obtained from this research, it showed variations in tooth position and vertical dimension related to the RP technique. This may relate to un-polymerized resins used for manufacturing the printed dentures that require an additional final light-polymerization step to complete the progress. Prostheses deformation may take place when the partially polymerized complete denture is demounting from the build platform. Besides, a residual layer of un-polymerized resin continually remains on the finished prostheses. ${ }^{19}$

Inaccuracies of the RP strategy can be incorporated into each step, involving design in the CAD software, slicing in the printing software, and the printing procedure itself. Many factors determine the precision of the printed object, like light intensity, orientation, and " angle of printing, number of layers, the used software, layer shrinkage, amount of supporting structure, and post-processing steps. ${ }^{20}$

At the time of polymerization, the heat-curing resin tends to tilt the artificial teeth. Once the resin is removed from the flask, releasing of residual stresses that occur at the time of packing took place, which contributes to resin shrinkage. In addition to differential thermal contraction during flask cooling, strain accompanying stress release throughout deflasking, water/ powder ratio of investing gypsum. Setting expansion of gypsum during investing causes linear alterations, which are presented as a causative factor for the denture base poor fit and artificial teeth displacement. Subsequently, alteration in the vertical dimension. ${ }^{21,22}$

No technique can produce zero movements of the tooth; however, various degrees of movement of the tooth were observable and found to be statistically crucial between the tested techniques. Thus, in this study, the three-dimensional printed monolithic group showed the least dimensional changes reflected by decreased overall denture teeth deviation and vertical dimensional changes. This is consistent with Goodacre et al., who documented that pressure-requiring processing techniques showed more occlusal movement of the tooth than those requiring no pressure. They also stated that the technique that produces the least tooth movement and denture base distortion is considered to be superior in processing technology. ${ }^{23}$

\section{CONCLUSION}

Under the restrictions of this research, tooth movements occur to some degree in both surveyed groups. However, three-dimensional monolithic printing dentures displayed greater dimensional precision with respect to denture teeth deviation and vertical dimensional alteration compared to the heat-curing acrylic resin.

\section{REFERENCES}

1. Sandra Lúcia, Andrade de Freitas, William Cunha Brandt, et al: Effect of Thermocycling, Teeth, and Polymerization Methods on Bond Strength Teeth-Denture Base. Int J Dent. 2018;4;15

2. Savirmath A.: A Comparative Evaluation of the Linear Dimensional Changes of Two Different Commercially Available Heat Cure Acrylic Resins during Three Different Cooling Regimens. J ClinDiagn Res. 2016; 10(11): ZC50-ZC54

3. Sayed ME, Swaid SM, PorwalA. :Effect of Cast Modification on Linear Dimensional Change of Acrylic Tooth Position Following Maxillary Complete Denture Processing. J Prosthodont. 2017; 26(8):659-663.

4. Abby A, Kumar R, Shibu J., et al : Comparison of the linear dimensional accuracy of denture bases cured the by conventional method and by the new press technique.Indian J Dent Res. 2011;22(2):200-204.

5. Pachauri P, Sangur R, Bathala LR, et al :.Comparison of the linear dimensional accuracy of the maxillary denture teeth by three flask closure methods. An in vitro study. Odontostomatol Trop. 2016;39(153):14-22. 
6. Savabi G, Savabi O, Dastgheib B, et al.: Effect of the processing cycle on dimensional changes of heatpolymerized denture base resins.Dent Res J (Isfahan). 2015;12(4):301-306.

7. Hyun-Ji Hwang, Sang J L, Eun-Jin Park ,et al : Assessment of the trueness and tissue surface adaptation of CADCAM maxillary denture bases manufactured using digital light processing. J Prosthet Dent .2019;121(1):110-117

8. Abid Haleem and Mohd Javaid.: Polyether ether ketone (PEEK) and its manufacturing of customised 3D printed dentistry parts using additive manufacturing. ClinEpidemiol Glob Health. 2019 https://doi.org/10.1016/j.cegh.2019.03.001

9. Akinori Tasaka, Satoru Matsunag, KentoOdaka.: Accuracy and retention of denture base fabricated by heat curing and additive manufacturing. journal of prosthodontic research. 2019;63:85-89

10. Totu E E, Nechifor A C, Nechifor G,et al :. Poly(methyl methacrylate) with $\mathrm{TiO} 2$ nanoparticles inclusion for stereolitographic complete denture manufacturing _ the fututre in dental care for elderly edentulous patients?. J of Dent. 2017;59: 68-77

11. Charan J, Biswas T. : How to Calculate Sample Size for Different Study Designs in Medical Research? Indian J Psychol Med. 2013: 35(2): 121-126.

12. Basso MFM, Nogueira SS, Arioli-Filho JN.: Comparison of the occlusal vertical dimension after processing complete dentures made with lingualized balanced occlusion and conventional balanced occlusion. J Prosthet Dent 2006; 96(3):200-204.

13. Lee MJ, Kim MJ, Oh SH, Kwon JS.: Novel Dental Poly (Methyl Methacrylate) Containing Phytoncide for Antifungal Effect and Inhibition of Oral Multispecies Biofilm. Materials (Basel). 2020;13(2).pii: E371

14. Vojdani M, Giti R.: Polyamide as a Denture Base Material: A Literature Review.J Dent (Shiraz). 2015 ;16: 1-9.
15. Slaviero TV, Simon GH, Tagliari I, et al : Effect of polymerization techniques on vertical dimension and tooth position in complete dentures. ActaOdontol Latinoam. 2011; 24(2):211-217

16. Babu S, Manjunath S, Vajawat M.: Effect of palatal form on movement of teeth during processing of complete denture prosthesis: An in-vitro study.Contemp Clin Dent. 2016;7(1):36-40

17. Sotto-Maior BS, Jóia FA, Meloto CB, et al: Effect of double flasking and investing methods on artificial teeth movement in complete dentures processing.Gerodontology. 2012;29(2):e435-9.

18. Javaid M, Haleem A, Kumar L. :Current status and applications of 3D scanning in dentistry. Clin Epidem and Global Health.2019; 7: 228-233.

19. Slaviero TV, Simon GH, Tagliari I,et al :. CAD-CAM milled versus rapidly prototyped (3D-printed) complete dentures: An in vitro evaluation of trueness. J Prosthet Dent. 2019;121:637-43

20. Lee S, Hong SJ, Paek J, Pae A, Kwon KR, Noh K. Comparing accuracy of denture bases fabricated by injection molding, CAD/CAM milling, and rapid prototyping method.J AdvProsthodont. 2019 Feb;11(1):55-64

21. Dos Santos MB, Consani RL, Mesquita MF.:Influence of different metal flask systems on tooth displacement in complete upper dentures.Gerodontology. 2012;29(1):30-5

22. Consani RL, Mesquita MF, Sobrinho LC, et al:. Dimensional accuracy of upper complete denture bases: the effect of metallic flask closure methods. Gerodontology 2009; 26: 58- 64

23. Goodacre B J, Goodacre C J, Baba N Z,et al:. Comparison of denture tooth movement between CAD-CAM and conventional fabrication techniques. JProsthet Dent .2018;119:108-115 